\title{
Demographic Data
}

Please provide the following anonymous demographic data, which will aid the analysis of your results.

1. Gender:

Female

Male

Other :

2. Date of birth (YYYY-MM-DD):

YYYY-MM-DD

3. People in Canada come from many racial or cultural groups. You may belong to more than one group on the following list. Are you:

White

Chinese

South Asian

Black

Filipino

Latin American

Southeast Asian

Arab

West Asian

Japanese

Korean

Aboriginal

Other

4. Select all languages you can communicate in (speak, understand, read, and write), along with the level of fluency for each:

Language

English

\section{Fluency}

\author{
Native \\ Fluent \\ Intermediate
}

Beginner 
French

Native

Fluent

Intermediate

Beginner

Select another language...

$\boldsymbol{\nabla}$

Native

Fluent

Intermediate

Beginner

Add Another Language

5. Education level (highest completed, or currently being completed)

University - Doctoral Degree

University - Master's Degree

University - Undergraduate Degree

College

High School

Elementary School

None

6. Have you ever been diagnosed with dementia? Please select any type of dementia with which you've been diagnosed. If you've never been diagnosed, please leave this section blank:

Alzheimer's disease (AD)

Vascular dementia (VD)

Mild cognitive impairment (MCl)

Dementia with Lewy Bodies (DLB)

Frontotemporal dementia (FTD)

Mixed dementia

Parkinson's disease (PD)

Other :

7. Do you currently take any medications for dementia?

Yes

No

8. Have you been a regular smoker (of tobacco) within the last 3 years? 
No

9. Country you were born in:

Select country...

10. Country you currently reside in:

Select country...

Submit

(C) University of Toronto 2018 